Canadian Science Publishing

Biochemistry and Cell Biology Biochimie et biologie cellulaire

\title{
Regulation of expression of the p21CIP1 gene by the transcription factor ZNF217 and MDM2
}

\begin{tabular}{|r|l|}
\hline Journal: & Biochemistry and Cell Biology \\
\hline Manuscript ID & bcb-2016-0026.R1 \\
\hline Manuscript Type: & Article \\
\hline Date Submitted by the Author: & 12-Apr-2016 \\
\hline Komplete List of Authors: & $\begin{array}{l}\text { Mantsou, Aglaia; Aristotle University of Thessaloniki Faculty of Sciences } \\
\text { Koutsogiannouli, Evangelia; Aristotle University of Thessaloniki School of } \\
\text { Medicine } \\
\text { Haitoglu, Konstantinos; Aristotle University of Thessaloniki School of } \\
\text { Medicine, Lab Biological Chemistry } \\
\text { Papavassiliou, Athanasios; UNIVERSITY OF ATHENS MEDICAL SCHOOL, } \\
\text { Biological Chemistry } \\
\text { Papanikolaou, Nikolaos; Aristotle University of Thessaloniki, Medicine }\end{array}$ \\
\hline Keyword: & \begin{tabular}{l} 
affinity chromatography, MDM2, ZNF217, p21 promoter \\
\hline
\end{tabular} \\
\hline
\end{tabular}




\section{Regulation of expression of the $p 21^{C I P 1}$ gene by the}

\section{transcription factor ZNF217 and MDM2}

Aglaia Mantsou, Evangelia Koutsogiannouli*, Costas Haitoglou, Athanasios

G. Papavassiliou, and Nikolaos A. Papanikolaou

Aglaia Mantsou, Costas Haitoglou and Nikolaos A. Papanikolaou: Laboratory of

Biological Chemistry, Division of Biological Sciences and Preventive Medicine, Faculty

of Medicine, Aristotle University of Thessaloniki, University Campus Bldg 16a, 54124

Thessaloniki, Greece.

Athanasios G. Papavassiliou: Department of Biological Chemistry, Medical School,

National and Kapodistrian University of Athens, 75 M. Asias Street, 11527 Athens,

Greece.

Corresponding author: Nikolaos A. Papanikolaou (email: papanikn@med.auth.gr)

*Current position: Post-doctoral fellow, Department of Urology, Heinrich Heine

University, Dusseldorf, Germany 


\begin{abstract}
:
Using MDM2 protein-specific affinity chromatography and mass spectrometry, we have isolated the protein product of the oncogene, $Z N F 217$, a transcription factor and a component of a HelaS-derived HDAC1 complex, as a novel MDM2-interacting protein. When co-expressed in cultured cancer cells, ZNF217 forms a complex with MDM2 and its ectopic over-expression reduces the steady-state levels of acetylated p53 in cell lines suppressing its ability to activate expression of a p21 promoter construct. In silico analysis of the p21 promoter revealed the presence of several ZNF217-binding sites. These findings suggest that MDM2 controls p21 expression by at least two mechanisms: Through ZNF217-mediated recruitment of HDAC1/MDM2 activity which inhibits p53 acetylation and through direct interaction with its binding site(s) on the p21 promoter.
\end{abstract}

Keywords: Affinity chromatography, MDM2, p21 promoter, ZNF217

\begin{abstract}
Abbreviations
MDM2: mouse double minute 2, ZNF217: zinc finger 217, CoREST: corepressor of RE1 silencing transcription factor/neural restrictive silencing factor, HDAC: histone deacetylase, CBP: CREB-binding protein, TSA: trichostatin A, LLnV: carbobenzoxy-Lleucyl-L-leucyl-L-norvalinal, PID: p53 inhibitor of degradation, NuRD: nucleosome remodeling and deacetylase complex, $\mathrm{p} 21^{\text {cipl }}$ : cyclin inhibitor 1 (protein 21$)$, MTA1/2: metastasis associated antigen 1/2, GST: glutathione S transferase, DTT: dithiothreitol, PMSF: phenyl methyl sulfonyl fluoride, DOC: deoxycholate, SUV39H1: suppressor of
\end{abstract}


ARTICLE

variegation 39H1, PARP: polyadenylate ribosyl phosphorylase, EDTA: ethylene diamine triacetic acid 


\section{Introduction}

MDM2 is encoded by the murine double minute clone 2 ( $m d m 2$ ) locus and is the main intracellular regulator of the p53 tumor suppressor protein (reviewed in Prives 1998; Woods and Vousden 2001). MDM2 co-immunoprecipitates with a temperaturesensitive rat p53 mutant at the permissive temperature (Cahilli-Snyder et al. 1987) and its over-expression in cell lines prevents p53-mediated transactivation of a reporter gene under the control of a promoter bearing a p53-responsive element as well the in vivo expression of the $p 21^{C I P}$ gene, which is a cell cycle regulator (Momand et al. 1992), underscoring the critical role of MDM2 in p53 functional control.

MDM2 controls the function of the p53 protein primarily by regulating its stability through ubiquitination. Attachment of ubiquitin marks proteins for rapid proteolytic degradation via the $26 \mathrm{~S}$ proteasome pathway in the cytoplasm. In unstressed mammalian cells, MDM2 interacts with the activation domain of p53 at its N-terminus with high affinity and acts as an ubiquitin ligase. It attaches the 76-amino acid protein ubiquitin to free lysine residues at positions $370,372,373,381$ and 382 of the p53 Cterminus (Honda et al. 1997; Honda et al 2000) and ultimately it shuttles it to the cytoplasm. Interestingly, the same positions are acetylated by $\mathrm{p} 300 / \mathrm{CBP}$ after genotoxic stress and methylated by SMYD2 and SET9, implying that these modifications are functionally reciprocally related (Ito et al. 2001).

The p53 tumor suppressor protein is critical to cellular pathways that maintain genomic integrity and is normally present at undetectable levels in most unstressed cells. Following genotoxic stress signals, the p53 protein is stabilized and activated as a transcription factor by covalent modifications, ultimately leading to cell-cycle inhibition 
and growth arrest or apoptosis (Woods, 2001 \#174). Acetylation of the p53 protein by genotoxic stress correlates well with its activation as a transcription factor and consequently with its function as a tumor suppressor, which ultimately induces genetic programs of cell-cycle arrest and/or apoptosis. Deacetylation of p53 and hence its inactivation is equally important and it involves association with active deacetylase (HDAC) complexes.

Several experimental observations suggest that MDM2 also controls p53 function by non-ubiquitylation mechanisms that regulate p53 acetylation/methylation. First, overexpression of MDM2 efficiently inhibits acetylation of p53 in the presence of TSA, an HDAC-specific inhibitor, and in a dosage-dependent manner by coexpressed p300 without affecting the levels of either $\mathrm{p} 300$ or p53, suggesting that the inhibition is a direct effect of MDM2 (Kobet et al 2000). Second, stabilization of p53 by treatment with the proteasome inhibitor $\mathrm{LLnV}$ fails to induce a concomitant increase in acetylation by coexpressed p300 in the presence of MDM2, suggesting that MDM2 actively suppresses this modification in the presence of over-expressed p300 (Ito et al. 2001). Third, addition of TSA effectively reverses the MDM2-mediated inhibition of p53 acetylation, indicating that MDM2 is recruiting HDAC activity to deacetylate p53. Fourth, MDM2 binds to the transactivation domain of p53 and displaces coactivators and histone acetyltransferase activity (Teufel et al 2007). Fifth, the protein SMAR1 interacts with phosphorylated p53 at Ser15 and with MDM2 forming a ternary complex resulting in deacetylation of p53 and in suppression of p21 promoter activation (Pavithra et al 2009). Sixth, it was demonstrated that the p53-binding mutant of MDM2 ( $\Delta 58-92)$ and the p300-binding mutant $(\Delta 199-222)$ were ineffective at inhibiting p53 acetylation and also were inactive 
at degrading it. In contrast, the RING domain of MDM2 is completely dispensable for inhibition of p53 acetylation. MDM2 is known to recruit methyltransferase complexes containing SUV39H1 and EHMT1 to p53 and methylates H3-K9 in vivo reducing its activity (Chen et al 2010). Lastly, the fraction of acetylated p53 is substantially elevated in cells treated with TSA (Sakaguchi et al. 1998).

The ability of p53 to activate programs of cell death or arrest resulting from genotoxic stress implies that under normal, i.e. unstressed, cellular conditions its activity is tightly but flexibly and rapidly regulated by acetylation/deacetylation cycles. Deacetylation is, therefore, a critical reverse component in the regulation of p53 function. P53 is known to associate with different HDAC enzyme complexes. Several HDACcontaining complexes have been purified to date and their components identified ( $\mathrm{Ng}$ and Bird, 2000). Notably, PID/MTA2, a component of the nucleosome remodeling and deacetylation protein complex (NuRD) (Zhang et al. 1998) directly interacts and deacetylates $\mathrm{p} 53$ by recruiting $\mathrm{HDAC} 1 / 2$, and it inhibits its tumor suppressor function (Luo et al., 2000). Deacetylase inhibitors cause cell death by stabilizing p53 acetylation, leading to cell cycle arrest and death through activation of $p 21^{C I P}$ expression $(\mathrm{Oh}$ et al, 2012) and/or by inhibiting MDM2 expression (Sonnemann et al. 2014). Lastly, overexpression of HDACs leads to inhibition of the transcriptional activity of p53 (Juan et al. 2000), supporting the notion that HDAC activity is critical for attenuation of p53 function. Our aim in this work was to isolate and characterize novel MDM2 interactors in deacetylase complexes that are important for $\mathrm{p} 53 / \mathrm{p} 21$ function, and to begin dissecting their regulatory roles. 


\section{Materials and Methods}

\section{Preparation of plasmids}

The full length and truncated versions of GST-MDM2 (amino acids 1-110, 110-280, 1220 and 280-491 respectively) were inserted by PCR as NdeI/BamHI fragments into pGEX-2TL (Pharmacia, USA). Full length ZNF217 was inserted by PCR into vector FLAG-pCIN4 as an NdeI-NotI fragment. For stable FLAG-ZNF217 expression, H1299 lines were prepared with G418 selection according to Li et al, 2004, using pCIN43XFLAG-ZNF217 inserted by PCR as an NdeI/Not1 fragment from a pBJ5-ZNF217 plasmid (You et al, 2001).

\section{Construction of GST-MDM2 affinity columns and protein-affinity purification of proteins interacting with MDM2}

In order to purify proteins in HDAC1 complexes that interact with full length or truncated MDM2, deletion human MDM2 cDNAs were amplified by PCR and subcloned into pGEX-2TL vectors (Pharmacia, USA). The corresponding recombinant GSTfusion proteins were expressed in BL21 E. coli cells, extracted with FLAG lysis buffer (20 mM Tris/HEPES, pH 8.0, 0.5 mM EDTA, 20\% glycerol, $1 \mathrm{M} \mathrm{NaCl,} \mathrm{1 \%} \mathrm{Triton-100,}$ 0.5 mM PMSF, $1 \mathrm{mM}$ DTT) and purified on glutathione-Sepharose (Sigma, USA). The purified fusion GST-MDM2 proteins were used as ligands for affinity chromatography with FLAG-HDAC1 protein complexes that were affinity purified with FLAG peptide from stably expressing HeLaS cell extracts. Affinity columns were prepared by packing 40-80 $\mu \mathrm{l}$ of GST or GST- MDM2 proteins into 1-200 $\mu$ l pipet tips and gravity 
equilibrated with buffer ( $0.1 \mathrm{M} \mathrm{NaCl}, 20 \mathrm{mM}$ Tris/Hepes, pH 8.0, $0.5 \mathrm{mM}$ EDTA, 20\% glycerol, 1\% Triton-100, 0.5 mM PMSF, 1 mM DTT).

Prior to loading of the HDAC1 complex, the MDM2 columns were equilibrated with $1 \mathrm{M} \mathrm{NaCl} / 1 \%$ deoxycholate-DOC (high salt buffer) and in order to clean them from debris. Purification was carried out by loading $300 \mu \mathrm{l}$ aliquots of immunopurified HelaSexpressed FLAG-HDAC1 complex. The bound complexes were washed three times with $0.1 \mathrm{M} \mathrm{NaCl} / 0.1 \% \mathrm{NP}-40$ buffer to dissociate weakly and non-specific bound proteins and eluted three times with high salt buffer. One third of the eluted fractions were subjected to silver staining and the rest to western blot analysis to identify the interacting proteins (Harlow, 1999).

\section{Electrophoresis and silver staining}

Fractions were obtained by elution as described in the text and $15 \mu 1$ of eluted sample was electrophoresed at $150 \mathrm{~V}$ for $6 \mathrm{~h}$. After elution from the MDM2-specific column, aliquots of 10-20 $\mu 1$ were electrophoresed onto 5-17\% gradient polyacrylamide gels and stained with silver nitrate to reveal proteins retained by the columns. Buffer was used in control affinity experiments.

\section{Analysis of MDM2 and ZNF217 interactions in cultured cancer cells}

For in vivo interactions, H1299 cells in $10 \mathrm{~cm}$ dishes (Corning) were transiently transfected with $5 \mu \mathrm{g}$ of MDM2 or $10 \mu \mathrm{g}$ of FLAG-ZNF217 plasmids, harvested with 0.1 $\mathrm{M} \mathrm{NaCl} / 0.1 \%$ deoxycholate, immunoprecipitated with M2 anti-FLAG antibody (Sigma, USA) conjugated to agarose resin, washed and eluted with FLAG peptide (Sigma, USA). Fifty (50) $\mu \mathrm{g}$ of input lysate and immunoprecipitated fractions were electrophoresed in 
7\% gel, immunoblotted with anti-MDM2 monoclonal antibody (Santa-Cruz, USA), stripped and re-blotted with anti- FLAG, M2 monoclonal antibody (Sigma, USA).

\section{Analysis of p53 acetylation in cultures cancer cells}

H1299 cells stably expressing a pCIN4-FLAG-ZNF217 fusion protein were transfected with $5 \mu \mathrm{g}$ of CMV-p53, or $5 \mu \mathrm{g}$ of PARP-encoding plasmids along with $1 \mu \mathrm{g}$ of GFP plasmid as an internal expression control, using a standard calcium phosphate method (Molecular Cloning: A Laboratory Manual, $4^{\text {th }}$ Edition, 2012). Twenty four hours after transfection, total cell extracts were purified with lysis buffer $[25 \mathrm{mM}$ Tris-Cl, $\mathrm{pH}$ 8.0, $25 \mathrm{mM}$ HEPES, $\mathrm{pH}$ 8.0, $137 \mathrm{mM} \mathrm{NaCl}, 1 \%$ TritonX-100, 0.2\% sarcosyl, $10 \%$ glycerol, $1 \mathrm{mM}$ EDTA, pH 8.0, 1mM NaF, $1 \mathrm{mM}$ sodium orthovanadate, $1 \mathrm{mM}$ PMSF1 mM DTT and protease inhibitor cocktail (Sigma, USA)] and subjected to immunoblot analysis with anti-acetyl p53 polyclonal antibody (PAb Lys(Ac)-C; Luo et al. 2000). The membrane was also probed with anti-GFP monoclonal antibody or DO-1 monoclonal antibody, which recognizes all forms of $\mathrm{p} 53$.

\section{Analysis of p53-mediated activation of a p21-luciferase promoter}

For transcriptional activation analysis of the $p 21$ promoter, H1299 cells were transiently transfected with different amounts of p53, MDM2 and ZNF217, p21luciferase and load control RT-luciferase plasmid constructs in 6-well plates (Corning) for $18 \mathrm{~h}$, using the calcium phosphate method. They were then harvested and luciferase activity was measured according to the manufacturer's instructions (Promega, USA) in a Berthold luminometer (Berthold Sirius Single Tube Luminometer, Germany). 


\section{In silico analysis of the human p21 promoter for ZNF217 consensus binding sites}

Several thousand nucleotides of the human $C D K N 1 A$ promoter $\left(\mathrm{p} 21^{C I P 1}\right.$, NCBI Reference Sequence: NG_009364.1) along with the first exon were retrieved from the UCSC Genome Browser at https://genome.ucsc.edu and searched for the presence of consensus p53 and ZNF217 nucleotide binding sequences.

\section{Results}

MDM2 mutant $\triangle M D M 2(1-440)$ lacking the RING domain can suppress the ability of p53 to activate the p21 promoter

We tested the hypothesis that MDM2 might be acting through non-ubiquitylation mechanisms by assessing the ability of an MDM2 mutant ( $\triangle \mathrm{MDM} 2 / 1-440)$ lacking the C-terminal RING domain, which confers E3 ubiquitin ligase activity, or full length MDM2, to repress expression of a p21-luciferase promoter construct by co-transfected p53. As expected, successively increased amounts of transfected, full length MDM2 plasmid repressed the expression of the p21 promoter nearly completely (Figure 1A, light grey-blue bars). In contrast, the plasmid encoding $\triangle \mathrm{MDM} 2 / 1-440$ repressed the $\mathrm{p} 21$ promoter construct less effectively than full-length MDM2 protein (Figure 1A, purplereddish bars) suggesting that the RING domain is required for full repression but more importantly that $\triangle \mathrm{MDM} 2$ lacking its ubiquitylation activity can still repress p53 function and can engage in interactions with p53. Notably, MDM2 is known to recruit deacetylase/methylase complexes in regulating p53 function such as for example the methylase SUV39H1 (Chen et al. 2010), independent of its ubiquitylation activity, regulating its ability to function as a transcription factor. Moreover, the complexity of 
composition of $\mathrm{HDAC} 1 / 2$ complexes suggests that MDM2 is engaged in several interactions with different complexes.

\section{MDM2 interacts with the product of the oncogene ZNF217}

We searched for proteins in FLAG-HDAC1 deacetylase complexes, which might interact with MDM2, isolated by FLAG affinity chromatography from HeLaS cell extracts expressing a FLAG-HDAC1 construct, using MDM2 -specific, protein affinity columns. To this end, we cloned several deletion human MDM2 mutants (Figure 1B) into pGEX2TL vectors as GST fusion polypeptides lacking the C-terminal RING domain of MDM2, in order to avoid interactions with the RING domain, and used them as affinity columns (for overall strategy see Figure 1C). $\triangle \mathrm{MDM} 2(280-491)$ which contains the RING domain was used as an additional control for interactors. We chose HDAC1 because it is invariably present in most deacetylase complexes and because it is the most extensively studied deacetylase. It is part of the core histone deacetylase complex which consists of HDAC1, HDAC2, RBBP4 and RBBP7. The core complex can associate and form complexes with the proteins MTA2, MBD2, MBD3, MTA1L1, CHD3 and CHD4 to form the nucleosome remodeling and histone deacetylation (NuRD) complex, or with SIN3, SAP18 and SAP30 to form the SIN3 HDAC complex.

In several experiments with three consecutive elutions two protein bands with molecular weights between 110 to $115 \mathrm{kD}$ were consistently retained and enriched by the GST- $\triangle$ MDM2 (1-110) column (Figure 2A, lanes 2 and 8) representing amino terminal amino acids $1-110$ and less so by the GST- $\triangle$ MDM2 (1-220) column. These proteins were absent in GST control columns (Figure 2A, lanes 10, 11), in the buffer (B) control 


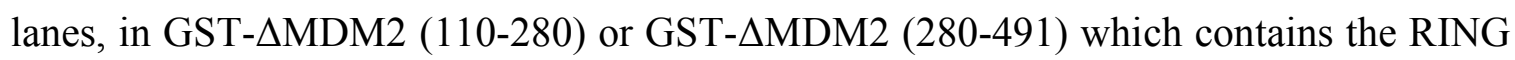
domain (lanes 4-7). Following a large preparation, the bands retained by MDM2 (1-110) were excised and subjected to mass spectrometry; a total of 7 peptides for p110 and 6 peptides for p115 were obtained. All peptides were derived from the putative transcription factor ZNF217 encoded by the complex locus znf217 (GenBank accession number NM_006526) which is located at 20q13.2. This locus apparently encodes several proteins(http://www.ncbi.nlm.nih.gov/ieb/research/acembly/av.cgi?db=human\&term=ZN F217\&submit=Go) with no sequence overlap. The experimentally detected protein is associated with diseases such as glioblastoma (Mao et al. 2011) and breast cancer (Krig et al. 2010; Thollet et al. 2010; Nonet et al. 2001). Also, ZNF217 is a component of a CoREST complex which has HDAC1 activity. The analysis with the MDM2 deletion mutants indicated that ZNF217 interacted with the N-terminus of MDM2 (Figure 2A, lane 2). We confirmed the presence of the core components HDAC1, CHD3 and RBBP7with immunoblot analysis of the same samples (Figure 2A, right panel).

\section{MDM2 and ZNF217 interact when co-expressed in cultured cell lines}

The retention of a ZNF217/HDAC1 complex by protein-specific affinity column $\triangle \mathrm{MDM} 2$ (1-110) suggested that the two proteins might also interact when co-expressed in cell lines. In order to determine if ZNF217 physically interacts with co-expressed MDM2, plasmids encoding an N-terminally FLAG-tagged full length ZNF217 and nontagged MDM2 driven by the CMV promoter, were co-transfected into H1299 cells and whole cell extracts were immunoprecipitated with resin conjugated to anti-FLAG antibody, eluted with FLAG peptide and immunoblotted for MDM2 with an anti-MDM2 monoclonal antibody (Figure 2B, top panel) or an anti- FLAG monoclonal antibody to 
verify ZNF217 expression (Figure 2B, middle panel or with a polyclonal anti-actin antibody (lower panel) as control. Both MDM2 and FLAG-ZNF217 were expressed (lanes 2, 3) and ZNF217 co-precipitated, albeit weakly, with MDM2 in H1299 cells (Lane 6), strongly supporting the hypothesis that the two proteins can form an intracellular complex.

\section{ZNF217 expression abrogates p53 acetylation in mammalian cells}

P300/CBP can directly acetylate p53 both in vitro and in vivo and it enhances the ability of p53 to (i) interact with its cognate DNA-binding sequences in the promoters of target genes such as the cell-cycle regulator p21, and (ii) to activate such promoters $(\mathrm{Gu}$ and Roeder 1997a,1997b). Because ZNF217 is a component of different HDAC1/2 complexes and is retained by the MDM2 affinity column and because MDM2 can effectively inhibit p300-mediated p53 acetylation, we also tested for its ability to inhibit the acetylation of p53 in H1299 cells stably expressing the FLAG-ZNF217 fusion protein. Plasmids encoding cDNAs for p53, FLAG- ZNF217 or PARP as a negative control (Figure 2C, lane 1) were co-transfected and the steady state levels of acetylated p53 were monitored using western blotting with a specific, well-characterized anti-acetyl p53 specific polyclonal antibody (Luo et al. 2001). As indicated in Figure 2C, overexpression of ZNF217 completely inhibited acetylation of p53 (lane 2). In contrast, expression of control vector pCIN4 did not affect p53 acetylation levels (lane 3). Expression of GFP control indicated that there were no significant differences in transfection efficiency. In addition, immunoblotting with the DO-1 anti-p53 antibody (a kind gift from Dr. Gu), which recognizes all forms of the protein, indicated that there were no differences in overall expression of $\mathrm{p} 53$, supporting the conclusion that the 
differences in p53 acetylation were due to specific inhibition rather than to profound differences in protein levels.

\section{ZNF217 expression inhibits p53-mediated activation of the p21 promoter}

Acetylation of p53 is a common posttranslational modification that is triggered by agents that can cause DNA damage (Ito et al. 2001). This modification has been well linked to p53 activation as a transcription factor, indicating that it may be a common requirement for $\mathrm{p} 53$ to activate target genes in growth or apoptosis. Deacetylation of p53 by recruited HDAC1/2 complexes (e.g. PID/MTA2) abrogates its transactivation activity towards the same promoter (Luo et al. 2001). Since ZNF217 can efficiently abrogate p53 acetylation, we first tested for its ability to regulate transactivation of the $p 21$ promoter, an important p53 target gene that is partly responsible for G1, p53-induced cell-cycle arrest. To that end, plasmids encoding p53, FLAG-ZNF217 and p21-luciferase were cotransfected into either H1299 or MEFp53(-/-) cells and the levels of luciferase were monitored using a Berthold luminometer. As indicated in Figure 3A in experiments with H1299 cells, over-expression of ZNF217 in H1299 cells efficiently reduced the levels of the p21-regulated luciferase reporter enzyme by p53 to near basal levels ( 9-fold decrease) (lane 3).

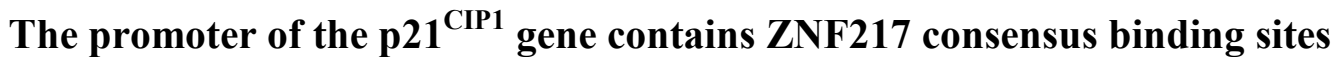

A report by Krig et al. 2007 (Table S6C) that siRNA-mediated down-regulation

of ZNF217 expression leads to activation of expression of $C D K N 1 A\left(p 21^{C I P 1}\right) \mathrm{mRNA}$ in cultured cells, suggested that ZNF217 actively suppresses $p 21^{C I P 1}$ expression. This prompted us to carefully scan the promoter of the $p 21^{C I P 1}$ gene for consensus ZNF217 
binding sites [CAGAA(C/G/T), see Krig et al. 2007 for details] and covering nucleotides roughly from -9600 to +3737 of the gene. We have uncovered several such consensus sites (Table 1, also see Figure 3B for an example of a ZNF217 binding site at bp position -3165 from the ATG start site and three helix turns downstream from a p53 site) suggesting that, in addition to inhibiting acetylation of $\mathrm{p} 53$ probably via interactions with MDM2 that lead to decreased p21 expression, ZNF217 could partly suppress expression of the $p 21^{C I P 1}$ gene via direct interactions with its binding sites on the promoter.

\section{Discussion}

In this study we report for the first time that the transcription factor ZNF217, a product of oncogene znf217, isolated from HeLaS cells in an HDAC1 multiprotein complex, is retained by an MDM2-specific affinity column ( $\triangle \mathrm{MDM} 2 / 1-110)$ and when co-expressed in cancer cell lines and regulates expression of a p21 promoter construct. ZNF217 forms HDAC1 deacetylase complexes which are usually recruited by transcription factors such as REST (You et al. 2001) or CtBP1/CtBP2/ HDAC2. Ectopic expression of ZNF217 in cancer cell lines can suppress the p53-mediated expression of a p21 promoter construct and can inhibit acetylation of p53 when ectopically expressed in cancer cell lines.

Acetylation of p53 is required for its activation as a transcription factor and for in vivo activation of expression of the $p 21$ gene which leads to cell-cycle arrest. When expressed in the same cancer cell lines, ZNF217 and MDM2 physically interact, albeit weakly, suggesting and they form intracellular complexes that may contain deacetylase activity (Figure 4, panel A). A truncated form of MDM2, $\triangle \mathrm{MDM} 2(1-440)$ that lacks the RING domain and a C-terminal fragment of ZNF217 synergize at inhibiting the p21 
promoter (Papanikolaou, unpublished) clearly implying that the RING domain-mediated ubiquitylation activity is dispensable for its interactions with ZNF217. In contrast, a form of ZNF217 that lacks the C-terminus cannot synergize with MDM2 at inhibiting the p21 promoter (Papanikolaou, unpublished). In vitro, we specifically demonstrate that ZNF217 that is present in an HDAC1 complex obtained from HeLaS cells that stably express a FLAG-HDAC1 construct can interact with the first 110 amino acids of MDM2 of the Nterminal domain (Figure 2A, lane 2). Interestingly, the SUVH1A methylase interacts with the acidic domain (Chen et al 2010) which however is absent in the $\triangle \mathrm{MDM} 2(1-110)$ fragment that we used in the affinity experiments implying that, just like ubiquitylation activity, methylase activity need not be present for this interaction to occur.

Using in silico analysis we demonstrate that, in addition to the p53 binding site, the p21 promoter contains ZNF217 consensus binding sequences (table 1). In particular, there is one ZNF217 and one p53 binding site located approximately 3165 nucleotides upstream on the proximal promoter. Notably, these binding sites on the $\mathrm{p} 21$ promoter are three helices apart from each other (Figure 3B) raising the possibility that ZNF217 might be competing with p53 complexes for binding on the p21 promoter. ZNF217 might displace p53 from its binding site by directly binding to its promoter site. Whether MDM2 is also required for this activity can be verified with chromatin immunoprecipitation experiments with anti-ZNF217 antibodies, followed by western blots for MDM2. Notably, ZNF217 represses expression of E-cadherin in vivo by direct binding to an identical binding motif on its promoter (Hayakawa and Nakayama 2011). Our experiments have demonstrated that expression of ZNF217 in cancer cells abrogates the acetylation of ectopically expressed p53 in the cancer cell line H1299 (Figure 2C) 
perhaps via interactions with MDM2. The ability of transfected, full length ZNF217 to nearly completely suppress p21 promoter activity, in comparison to truncated MDM2 which does so only partly, appears to be in agreement with its ability to both interact with MDM2 and deacetylate p53 and to repress the expression of the p21 promoter by directly binding to its cognate DNA site on the promoter.

Our data suggest that regulation of expression of the p21 promoter by ZNF217 might be mediated by two distinct, yet not mutually exclusive mechanisms (Figure 4). These mechanisms probably operate in addition to that of MDM2-mediated degradation of p53 (Grossman et al 1998; Geyer et al 2000). In the first mechanism, MDM2 recruits a multiprotein complex that contains ZNF217 and deacetylates p53, preventing the binding to its promoter site thus leading to inhibition of expression of the p21 promoter (Figure 4, panel A). Although weak, the interaction between ZNF217 and MDM2, when coexpressed in cancer cell lines suggests that additional polypeptides might be required. It is likely that such polypeptides are absent in the H1299 cells which, notably, lack p53 protein expression. Although we have not shown that p53 is part of a non-promoter bound MDM2/ZNF217 complex, we cannot exclude such a possibility. Indeed, the nearly complete abrogation of p53 acetylation (Figure 2C) by ectopically expressed ZNF217 in the cancer cell line, H1299, implies that p53 is probably part of the deacetylating complex. In the second mechanism, ZNF217 (with or without MDM2) recruits a multiprotein transcriptional repressor complex and binds directly to its cognate site(s) on the p21 promoter leading to suppression of its expression (Figure 4, panel B). Direct support for this mechanism can be provided by chromatin immunoprecipitation experiments using anti-ZNF217 antibodies and western blotting for MDM2. P53 need not 
be present in the recruited repressive transcriptional complex since ZNF217 can bind directly to its site on the promoter and displace bound p53 or prohibit its binding. Moreover, ZNF217 and MDM2 also do not need to interact for ZNF217 to bind directly to the promoter. It is likely that both mechanisms operate simultaneously, thus ensuring near complete repression of expression of the p21 promoter as shown in Figure 3A.

In conclusion, our data support two alternative, but not mutually exclusive mechanisms of regulation of the p21 promoter by ZNF217 and MDM2. In short-term regulation, over-expression of ZNF217 and MDM2 leads to complex formation with p53 which is deacetylated and thus prevented from interacting with its promoter DNA site (Figure 4, panel A). Our finding that MDM2 can directly interact with ZNF217 (via its N-terminus) when co-expressed in cancer cell lines, and can, therefore, recruit HDAC1/2 activity, raises the possibility that it can inhibit p53 acetylation directly in the short term thus preventing its binding to the promoter. Alternatively, though not exclusively, in long-term repression, ZNF217 recruits a transcriptional repressor complex and binds directly to its cognate site on the $\mathrm{p} 21$ promoter thus suppressing expression of the $\mathrm{p} 21$ gene (Figure 4, panel B).

\section{Acknowledgements}

This work was supported by 'Aristeia' (Excellence) award \# 90924-2013 from the Aristotle University of Thessaloniki to N.A. Papanikolaou. Initial experiments were performed at Columbia University (lab of Dr. W. Gu, Institute for Cancer Genetics) under a Kirschtein National Research Service Award to N.A. Papanikolaou. We are grateful to Drs. W. Gu for providing plasmid reagents and antibodies, S. Schreiber 
(Harvard University, Cambridge Massachusetts, USA) for a FLAG-HDAC1 construct and Jun Qin (Baylor College of Medicine, Houston Texas, USA) for MS analysis of peptides. 


\section{References}

Cahilly-Snyder L., Yang-Feng T., Francke U., and George D.L. 1987. Molecular analysis and chromosomal mapping of amplified genes isolated from a transformed mouse 3T3 cell line. Somat. Cell Mol Genet. 13(3):235-44.

Chen L, Li Z., Zwolinska AK.., Smith MA., Cross B., Koomen J., Yuan Z.M., Jenuwein T., Marine J.C., Wright K.L., and Chen J. 2010. MDM2 recruitment of lysine methyltransferases regulates p53 transcriptional output. EMBO J. 29(15):2538-52.

Geyer R.K., Yu Z.K., and Maki C.G. 2000. The MDM2 RING-finger domain is required to promote p53 nuclear export. Nat Cell Biol. 2(9):569-73.

Grossman S.R., Perez M., Kung A.L., Joseph M., Mansur C., Xiao Z.X., Kumar S., Howley P.M., and Livingston D.M. 1998. p300/MDM2 complexes participate in MDM2-mediated p53 degradation. Mol Cell. 4:405-15.

Gu, W., Shi, X.L., Roeder R.G. 1997a. Synergistic activation of transcription by CBP and p53. Nature 19: 387(6635):819-23.

Gu, W., and Roeder, R.G. 1997b. Activation of p53 sequence-specific DNA binding by acetylation of the p53 C-terminal domain. Cell 90 (4): 595-606.

Harlow E., and Lane D. 1999. Using Antibodies. A Laboratory Manual. Cold Spring Harbor, New York: Cold Spring Harbor Laboratory. 
Hayakawa T., Nakayama J. 2011. Physiological roles of class I HDAC complex and histone demethylase, J Biomed Biotechnol. doi: 10.1155/2011/129383.

Honda R., Tanaka H., Yasuda H. 1997. Oncoprotein MDM2 is a ubiquitin ligase E3 for tumor suppressor p53. FEBS Lett. 420(1):25-7.

Honda R., Yasuda H. 2000. Activity of MDM2, a ubiquitin ligase, toward p53 or itself is dependent on the RING finger domain of the ligase. Oncogene 19(11):1473-6.

Ito A, Lai C,H,, Zhao X, Saito S,, Hamilton M,H,, Appela E,, and Yao T.P. 2001. p300/CBP-mediated p53 acetylation is commonly induced by p53-activating agents and inhibited by MDM2. EMBO J. 20(6):1331-40.

Jin Y., Zeng S.X., Dai M.S., Yang X.J., and Lu H. 2002. MDM2 inhibits PCAF (p300/CREB-binding protein-associated factor)-mediated p53 acetylation. J Biol Chem. 277: 30838-30843.

Juan L.J., Shia W.J., Chen M.H., Yang W.M., Seto E., Lin Y.S., and Wu C.W. 2000. Histone deacetylases specifically down-regulate p53-dependent gene activation. J Biol Chem. 275(27):20436-43.

Kobet E., Zeng X., Zhu Y., Keller D., and Lu H. 2000. MDM2 inhibits p300-mediated p53 acetylation and activation by forming a ternary complex with the two proteins. Proc Natl Acad Sci U S A. 97(23):12547-52. 
Krig, S.R., Jin, V., X., Bieda, M.C., O'Geen, H., Yaswen, P., Green, R., and Farnham, P.J. 2007. Identification of genes directly regulated by the oncogene ZNF217 using chromatin immunoprecipitation (ChIP)-chip assays. J Biol Chem. 282(13): 9703-9712.

Li M., Chen D., Shiloh A., Luo J., Nikoaev A.Y., Qin J., and Gu W. 2002. Deubiquitination of p53 by HAUSP is an important pathway for p53 stabilization. Nature 461: 648-653.

Li M., Brooks C.L., Kon N., and Gu W. 2004. A dynamic role of HAUSP in the p53MDM2 pathway. Mol Cell 13(6):879-86.

Luo, J., Su, F., Chen, D., Shiloh, A., and Gu, W. 2000. Deacetylation of p53 modulates its effect on cell growth and apoptosis. Nature 408: 377-381.

Luo J., Nikolaev A.Y., Imai S., Chen D., Su F., Shiloh A., Guarente L., and Gu W. 2001. Negative control of p53 by Sir2alpha promotes cell survival under stress. Cell 107(2):137-48.

Mao X.G, Yan M., Xue X.Y., Zhang X., Ren H.G., Guo G., Wang P., Zhang W., and Huo J.L. 2011. Overexpression of ZNF217 in glioblastoma contributes to the maintenance of glioma stem cells regulated by hypoxia-inducible factors. Lab Invest. 91(7):1068-78.

Momand J., Wu H.H., and Dasgupta G. 2000. MDM2: master regulator of the p53 tumor suppressor protein. Gene 242(1-2):15-29.

Ng, H.N., and Bird. A. 2000. Histone deacetylases: silencers for hire. TIBS 25(3):121-6. 
Nonet G.H, Stampfer M.R., Chin K., Gray J.W., Collins C.C., and Yaswen P. 2001. The ZNF217 gene amplified in breast cancers promotes immortalization of human mammary epithelial cells. Cancer Res. 61(4):1250-4.

Oh E.T., Park M.T., Choi B.H., Ro S., Choi E.K., Jeong S.Y., and Park H.J. 2012. Novel histone deacetylase inhibitor CG200745 induces clonogenic cell death by modulating acetylation of p53 in cancer cells. Invest New Drugs 30(2):435-42.

Pavithra L., Mukherjee S., Sreenath K, Kar S., Sakaguchi K., and Roy S, Chattopadhyay S. 2009. SMAR1 forms a ternary complex with p53-MDM2 and negatively regulates p53-mediated transcription. J Mol Biol. 388(4):691-702.

Prives C. Signaling to p53: Breaking the MDM2-p53 circuit. 1998. Cell 95: 5-8.

Sakaguchi K., Herrera J.E., Saito S., Miki T., Bustin M., Vassilev A., Anderson C.W., and Appela E. 1998. DNA damage activates p53 through a phosphorylationacetylation cascade. Genes Dev. 12(18):2831-41.

Sonnemann J., Marx C., Becker S., Wittig S., Palani C.D., Krämer O.H., and Beck J.F. 2014. p53-dependent and p53-independent anticancer effects of different histone deacetylase inhibitors. Br J Cancer 110(3):656-67.

Thollet A., Vendrell J.A., Payen L., Ghayad S.E., Ben Larbi S., Grisard E., Collins C., Villedieu M., and Cohen PA. 2010. ZNF217 confers resistance to the pro-apoptotic signals of paclitaxel and aberrant expression of Aurora-A in breast cancer cells. Mol Cancer 9: 291. 
Teufel D.P., Freund S.M., Bycroft M., and Fersht A.R. 2007. Four domains of p300 each bind tightly to a sequence spanning both transactivation subdomains of p53. Proc Natl Acad Sci USA 104(17):7009-14.

You A., Tong J.K., Grozinger C.M., Schreiber S.L.. 2000. CoREST is an integral component of the CoREST- human histone deacetylase complex. Proc Natl Acad Sci U S A. 98(4):1454-8.

Wade P.A. 2001. Transcriptional control at regulatory checkpoints by histone deacetylases: molecular connections between cancer and chromatin. Hum Mol Genet. 10(7):693-8.

Woods, W.B., and Vousden, K.H.. 2001. Regulation of p53 function. Experimental Cell Res. 264: 56-66.

Zhang, Y., Ng, H.H., Erdjument-Bromage, H., Tempst, P., Bird, A., and Reinberg, D. 1999. Analysis of the Nurd subunit reveals a histone deacetylase core complex and a connection with DNA methylation. 13(15):1924-35. 
Table 1. ZNF217 binding sites on the human p21 promoter

\begin{tabular}{|c|c|c|c|c|}
\hline Sequence & Nucleotide location & $\begin{array}{c}\text { Position } \\
\text { before } \\
\text { start site } \\
\text { (bp) }\end{array}$ & $\begin{array}{l}\text { Chromosomal } \\
\text { location (bp) }\end{array}$ & Chain \\
\hline TGCAGAAG & Promoter & -7879 & 36676223 & Antisense \\
\hline TGCAGAAG & Promoter & -9504 & 36674598 & Antisense \\
\hline AACAGAAC & $1^{\text {nd }}$ intron of $5^{\prime}$ UTR & -6686 & 36677416 & Antisense \\
\hline AACAGAAG & $2^{\text {nd }}$ exon of $5^{\prime}$ UTR & -6178 & 36677924 & Antisense \\
\hline AACAGAAG & $2^{\text {nd }}$ intron of $5^{\prime}$ UTR & -975 & 36683127 & Antisense \\
\hline TACAGAAG & $2^{\text {nd }}$ intron of $5^{\prime}$ UTR & -1875 & 36682227 & Antisense \\
\hline AACAGAAG & $2^{\text {nd }}$ intron of $5^{\prime}$ UTR & -6127 & 36677975 & Antisense \\
\hline AACAGAAT & intron of CDS & +1202 & 36685305 & Sense \\
\hline $\begin{array}{l}\text { AGCAGAAG } \\
\text { GGCAGAAT }\end{array}$ & $\begin{array}{l}\text { intron of CDS } \\
\text { Promoter }\end{array}$ & $\begin{array}{l}+1629 \\
-3165\end{array}$ & $\begin{array}{l}36685732 \\
36671460\end{array}$ & Antisense \\
\hline
\end{tabular}


Figure Captions

Fig. 1. (A) MDM2 lacking the RING domain can suppress the ability of p53 to activate the p21 promoter. H1299 cells in 6-well plates were transiently transfected with $50 \mathrm{ng}$ of p53, and with increasing amounts (50, 100, 250 and $500 \mathrm{ng}$, lanes 1,2, 3 and 4 respectively) of full-length MDM2 plasmid (gray-blue bars) or of $\triangle \mathrm{MDM} 2(1-440)$ which lacks its C-terminal RING domain (reddish bars), $100 \mathrm{ng}$ of p21-Luc and $100 \mathrm{ng}$ RT-Luc plasmid as an internal standard for luciferase expression, using the calcium phosphate method. The average of three experiments is shown. Statistical significance was $p<0.05$. (B) Schematic representation of the 491 amino acid full-length human MDM2 protein and of Truncated MDM2. (i) NLS, nuclear localization signal; ZF, zinc finger; RF, RING finger. Also shown are some of the factor interacting domains. (ii) Deletion MDM2 ( $\triangle \mathrm{MDM} 2)$ mutants used in the MDM2 affinity purification of ZNF217: cDNAs encoding the corresponding truncated proteins were cloned, expressed and purified from bacterial cells as described in Materials and Methods. Marker, (lane 1), MDM2 amino acids 1-110 (lane 2), 110-280 (lane 3), 280-491 (lane 4), 1-220 (lane 5) and control GST (lane 6). (C) Strategy for the MDM2 protein-specific affinity chromatography. The GST- $\triangle \mathrm{MDM} 2$ fragments shown in 1B were purified from $\mathrm{B} 121$ bacterial cells and were used for the isolation of MDM2-interacting proteins in HDAC1 complexes shown in Figure 2A as described in Materials and Methods.

Fig. 2. In vitro and in vivo Interactions between MDM2 and ZNF217. (A) Purification of ZNF217 from an HDAC1/2 complex by MDM2 protein-specific affinity chromatography. Left panel, silver-stained 5-17\% gradient SDS-PAGE gel of 
eluates of FLAG-HDAC1 complex. Fractions were obtained as described in Materials and Methods and $15 \mu \mathrm{l}$ of eluted sample was electrophoresed at $150 \mathrm{~V}$ for $6 \mathrm{~h}$. The gel was subsequently fixed and stained with silver nitrate. Shown are the two electrophoretic ZNF217 species, 110 and $115 \mathrm{kDa}$, that were retained be the MDM2 columns (amino acids 1-110 or 1-220) (lanes 2, 3), amino acids 110-280) (lanes 4, 5), amino acids 280491 (lanes 6, 7), amino acids 1-220 (lanes 8, 9) and GST as control for non-specific interactions (lanes 10,11). $\mathrm{NaCl} 100 \mathrm{mM} / 0.1 \%$ Triton $\mathrm{X}-100$ buffer was passed through the column as a control. The lower, heavy red arrows point to non-specific interactors. P110 and p115-derived peptides were analyzed by mass spectrometry. Two (2) $\mu 1$ of FLAG-HDAC1 affinity immunoprecipitate (IP) eluate was loaded as a control (lane 1). Also GST was used as a control for non-specific interactions (lanes 6, 7). M is for MDM2 and $\mathrm{B}$ is for buffer. Right panel, western blot confirmation of the presence of core HDAC1 complex components $\mathrm{CHD} 3$ and RBBP7 in the $\triangle \mathrm{MDM} 2(1-110)$ affinity retentate. (B) ZNF217 and MDM2 form an intracellular complex in H1299 cells: H1299 cells were transiently transfected with $5 \mu \mathrm{g}$ of MDM2 or $10 \mu \mathrm{g}$ of FLAGZNF217 plasmids, harvested with buffer $\mathrm{NaCl} 100 \mathrm{mM} / 0.1 \%$ Triton $\mathrm{X}-100$, immunoprecipitated with anti- FLAG antibody conjugated to agarose resin, washed and eluted with FLAG peptide. Fifty (50) $\mu \mathrm{g}$ of input lysates from FLAG vector-transfected whole cell lysate (lane 1), MDM2-transfected (lane 2) or co-transfected MDM2/FLAGZNF217 (lane 3) and anti-FLAG immunoprecipitated fractions (lanes 4-6) were electrophoresed in $7 \%$ gel, immunoblotted with an anti-MDM2 monoclonal antibody (upper panel). Then they were stripped and reblotted with an anti-FLAG monoclonal antibody (Sigma, M2 MAb) (lower panel). (C) Expression of ZNF217 reduces p53 
acetylation in mammalian cells. H1299 cells stably expressing 3XFLAG-ZNF217 fusion protein were transfected with $5 \mu \mathrm{g}$ of pCMV-p53, or $5 \mu \mathrm{g}$ of PARP encoding plasmids along with $1 \mu \mathrm{g}$ of GFP plasmid as an internal expression control. 24 hrs posttransfection, total cell extracts were purified with FLAG lysis buffer and subjected to immunoblot analysis with anti-acetyl p53 polyclonal antibody (upper panel) (PAb Lys(Ac)-C, Luo et al. 2000), anti GFP monoclonal antibody (lower panel) or DO-1 monclonal antibody (middle panel), which recognizes all forms of p53. Transfections with PARP (lane 1), ZNF217 (lane 2) or vector (lane 3).

Fig. 3. (A) Expression of ZNF217 abrogates the p53-mediated activation of the p21LUC promoter. H1299 cells in 6-well plates were transiently transfected with $50 \mathrm{ng}$ of p53, or $100 \mu \mathrm{g}$ of FLAG-ZNF217 plasmids, along with $100 \mathrm{ng}$ of p21-LUC plasmid as a reporter and $100 \mathrm{ng}$ of RT-LUC plasmid as an internal standard for luciferase expression, using the calcium phosphate method. Experiments were in triplicate and statistical significance was $<0.01$. (B) Part of the human p21cip1 (CDKN1A) promoter upstream from the start site are depicted. One consensus ZNF217-binding site (arrow, boxed on the right side) and a p53 binding site 3165 nucleotides upstream are shown. The dots between the starting codon and the rest of the sequence indicates promoter/5' UTR sequence not shown in the figure.

Fig. 4. Different models for ZNF217/MDM2/HDAC1-mediated repression of the p21 promoter. Panel A: In this model, MDM2/HDAC1/ZNF217 complexes deacetylate p53 prohibiting its binding to the $\mathrm{p} 53$ site on the $\mathrm{p} 21$ promoter and thus leading to repression 
of activation of p21 expression (short-term repression). The large $\mathrm{X}$ symbolizes the abrogation of p53 binding. The question mark symbolizes the fact that p53 was not shown to be part of an MDM/ZNF217 complex yet. Panel B: In this model, which is indirectly supported by the presence of ZNF217 sites on the p21 promoter, ZNF217 recruits a repression complex and binds directly to its cognate site(s) on the p21 promoter thus leading to inhibition of expression (long-term, transcriptional repression). 
